EFI-95-65

FERMILAB-PUB-95/345-T

ANL-HEP-PR-95-80

WIS-95/50-Oct.-Ph

hep-ph/9511363

November 1995

\title{
ANGULAR DISTRIBUTIONS AND LIFETIME DIFFERENCES IN $B_{s} \rightarrow J / \psi \phi$ DECAYS $]$
}

\author{
Amol S. Dighe \\ Isard Dunietz ${ }^{\beta}$ \\ Harry J. Lipkin回 回 \\ and \\ Jonathan L. Rosner ${ }^{2,3}$
}

\begin{abstract}
The strange $B$ meson $B_{s} \equiv \bar{b} s$ and its charge-conjugate $\bar{B}_{s} \equiv b \bar{s}$ are expected to mix with one another in such a way that the mass eigenstates $B_{s}^{H}$ ("heavy") and $B_{s}^{L}$ ("light") may have a perceptible lifetime difference of up to $40 \%$, with the CP-even eigenstate being shorter-lived. A simple transversity analysis permits one to separate the CP-even and CP-odd components of $B_{s} \rightarrow J / \psi \phi$, and thus to determine the lifetime difference. The utility of a similar analysis for $B^{0} \rightarrow J / \psi K^{* 0}$ is noted.
\end{abstract}

The Cabibbo-Kobayashi-Maskawa picture of weak charge-changing transitions [1] predicts the strange $B$ meson $B_{s} \equiv \bar{b} s$ and its charge-conjugate $\bar{B}_{s} \equiv b \bar{s}$ to mix with one another with a large amplitude. The mass eigenstates $B_{s}^{H}$ ("heavy") and $B_{s}^{L}$ ("light") with masses $m\left(B_{s}^{H}\right) \equiv m_{H}$ and $m\left(B_{s}^{L}\right) \equiv m_{L}$ are expected to be split by $\Delta m \equiv m_{H}-$ $m_{L} \approx 25 \bar{\Gamma}$, give or take a factor of two [2], where $\bar{\Gamma} \equiv\left(\Gamma_{H}+\Gamma_{L}\right) / 2 \approx \Gamma\left(B^{0}\right)\left(B^{0} \equiv \bar{b} d\right)$ and $\Gamma_{H, L} \equiv \Gamma\left(B_{s}^{H}, B_{s}^{L}\right)$. The measurement of such a large mass difference poses an experimental challenge.

To a good approximation, $\mathrm{CP}$ violation can be neglected in calculating the mass eigenstates, in which case they correspond to those $B_{s}^{( \pm)}$of even and odd $\mathrm{CP}$, with $B_{s}^{L}=B_{s}^{(+)}$and $B_{s}^{H}=B_{s}^{(-)}$as we shall see. The decay of a $\bar{B}_{s}$ meson via the quark

\footnotetext{
${ }^{1}$ To be submitted to Physics Letters B

${ }^{2}$ Enrico Fermi Institute and Department of Physics, University of Chicago, Chicago, IL 60637

${ }^{3}$ Theoretical Physics Division, Fermi National Accelerator Laboratory, Batavia, IL 60510

${ }^{4}$ High Energy Physics Division, Argonne National Laboratory, Argonne, IL 60439

${ }^{5}$ Department of Particle Physics, Weizmann Institute of Science, Rehovoth, Israel
} 
subprocess $b(\bar{s}) \rightarrow c \bar{c} s(\bar{s})$ gives rise to predominantly CP-even final states [3]. Thus the $\mathrm{CP}$-even eigenstate should have the greater decay rate. An explicit calculation [4] gives

$$
\frac{\Gamma\left(B_{s}^{(+)}\right)-\Gamma\left(B_{s}^{(-)}\right)}{\bar{\Gamma}} \simeq 0.18 \frac{f_{B_{s}}^{2}}{(200 \mathrm{MeV})^{2}}
$$

where $f_{B_{s}}$ is the $B_{s}$ decay constant (in a normalization in which $f_{\pi}=132 \mathrm{MeV}$ ). In one estimate [2], $f_{B_{s}}=225 \pm 40 \mathrm{MeV}$, while a compilation of lattice results [5] obtains $f_{B_{s}}=201 \pm 40 \mathrm{MeV}$ (90\% c.l. limits). The upper limit of $40 \%$ for (1) is based on an estimate of the maximum possible contribution from the $b(\bar{s}) \rightarrow c \bar{c} s(\bar{s})$ subprocess [6, 7].

The ratio of the mass splitting to the width difference of strange $B$ 's is predicted to be large and independent of CKM matrix elements [6, 8] (to lowest order, neglecting QCD corrections which may be appreciable):

$$
\frac{\Delta m}{\Delta \Gamma} \simeq-\frac{2}{3 \pi} \frac{m_{t}^{2} h\left(m_{t}^{2} / M_{W}^{2}\right)}{m_{b}^{2}}\left(1-\frac{8}{3} \frac{m_{c}^{2}}{m_{b}^{2}}\right)^{-1} \simeq-200,
$$

where $\Delta \Gamma \equiv \Gamma_{H}-\Gamma_{L}$. Here $h(x)$ decreases monotonically from 1 at $x=0$ to $1 / 4$ as $x \rightarrow \infty$; it is about 0.54 for $m_{t}=180 \mathrm{GeV} / c^{2}$. In view of the sign in Eq. (11) and since $\Delta m>0$ by definition, we then identify $B_{s}^{L}=B_{s}^{(+)}$and $B_{s}^{H}=B_{s}^{(-)}[6]$. If the mass difference $\Delta m$ turns out to be too large to measure at present because of the rapid frequency of $B_{s}-\bar{B}_{s}$ oscillations it entails, the width difference $\Delta \Gamma$ may be large enough to detect. The possibility of a value of $\Delta \Gamma / \Gamma$ for strange $B$ mesons large enough to measure experimentally has been stressed previously [3, 4, 9].

One can measure $\bar{\Gamma}$ using semileptonic decays, while the decays to $\mathrm{CP}$ eigenstates can be measured by studying the correlations between the polarization states of the vector mesons in $B_{s}^{( \pm)} \rightarrow J / \psi \phi$. (For similar methods applied to decays of other spinless mesons see, e.g., Ref. [10].) In the present note we describe a means by which the $J / \psi \phi$ final states of definite $\mathrm{CP}$ in $B_{s}$ decays may be separated from one another using a simple angular distribution based on a transversity variable [11, 12, 13]. This transversity variable allows one to directly separate the summed contribution of the even partial waves $(\mathrm{S}, \mathrm{D})$ from the odd one $(\mathrm{P})$ by means of their opposite parities. The CDF Collaboration [14] has recently reported the first angular distribution analysis of the decay $B_{s} \rightarrow J / \psi \phi$, obtaining a separation into longitudinal and transverse helicity amplitudes without making a statement yet about the CP-even and CP-odd contributions.

We summarize our main result. Consider the final state $J / \psi \phi \rightarrow \ell^{+} \ell^{-} K^{+} K^{-}$, where $\ell=e$ or $\mu$. In the rest frame of the $J / \psi$ let the direction of the $\phi$ define the $x$ axis. Let the plane of the $K^{+} K^{-}$system define the $y$ axis, with $p_{y}\left(K^{+}\right)>0$, so the normal to that plane defines the $z$ axis. (We assume a right-handed coordinate system.) We define the angle $\theta$ as the angle between the $\ell^{+}$and the $z$ axis. Then the time-dependent rate for the $J / \psi \phi$ mode is given by

$$
\frac{d^{2} \Gamma}{d \cos \theta d t}=\frac{3}{8} p(t)\left(1+\cos ^{2} \theta\right)+\frac{3}{4} m(t) \sin ^{2} \theta
$$




$$
=\frac{3}{8}[p(t)+2 m(t)]+\frac{3}{8}[p(t)-2 m(t)] \cos ^{2} \theta,
$$

where

$$
p(t)=p(0) e^{-\Gamma_{L} t} \quad(\mathrm{CP} \text { even }) \quad, \quad m(t)=m(0) e^{-\Gamma_{H} t} \quad(\mathrm{CP} \text { odd }) \quad,
$$

so that the probability of having a CP-even [CP-odd] state at proper time $t$ is given by $p(t) /(p(t)+m(t))[m(t) /(p(t)+m(t))]$. The angular distribution is normalized in such a way that

$$
\frac{d \Gamma}{d t}=\int_{-1}^{1} d(\cos \theta) \frac{d^{2} \Gamma}{d \cos \theta d t}=p(t)+m(t)
$$

As $t$ increases, one should see a growth of the $\sin ^{2} \theta$ component. The angle $\theta$ is an example of a transversity variable, whose utility for the determination of CP properties of multi-particle systems was pointed out some time ago [15.

The zero-angular-momentum states of two massive neutral vector mesons such as $J / \psi$ and $\phi$, both with the same CP (in this case, even) consist of two with even CP and one with odd CP. One can form states with orbital angular momenta $L=0$ (CP even), $L=1$ (CP odd), and $L=2$ (CP even).

Alternatively, one can decompose the decay amplitude $A$ into three independent components [16], corresponding to linear polarization states of the vector mesons which are either longitudinal (0), or transverse to their directions of motion and parallel (\|) or perpendicular $(\perp)$ to one another. The states 0 and $\|$ are P-even, while the state $\perp$ is $\mathrm{P}$-odd. Since $J / \psi$ and $\phi$ are both C-odd eigenstates, the properties under $\mathrm{P}$ are the same as those under CP.

Consider the polarization three-vectors $\boldsymbol{\epsilon}_{J / \psi}$ and $\boldsymbol{\epsilon}_{\phi}$ in the $J / \psi$ rest frame. The independent decay amplitudes are the rotationally invariant quantities linear in $\boldsymbol{\epsilon}_{J / \psi}^{*}$ and $\boldsymbol{\epsilon}_{\phi}^{*}$ and involving possible powers of $\hat{\mathbf{p}}$, a unit vector in the direction of the momentum of $\phi$ in the $J / \psi$ rest frame.

The two CP-even decay amplitudes are the combinations $\boldsymbol{\epsilon}_{J / \psi}^{*} \cdot \boldsymbol{\epsilon}_{\phi}^{*}$ (contributing to $A_{0}$ and $A_{\|}$) and $\boldsymbol{\epsilon}_{J / \psi}^{*} \cdot \hat{\mathbf{p}} \boldsymbol{\epsilon}_{\phi}^{*} \cdot \hat{\mathbf{p}}=\boldsymbol{\epsilon}_{J / \psi}^{* L} \boldsymbol{\epsilon}_{\phi}^{* L}$ (contributing only to $A_{0}$ ), where $\boldsymbol{\epsilon}^{L} \equiv \hat{\mathbf{p}} \cdot \boldsymbol{\epsilon}$. Equivalently, one can subtract off the longitudinal component of the polarization vectors to replace $\boldsymbol{\epsilon}_{J / \psi}^{*} \cdot \boldsymbol{\epsilon}_{\phi}^{*}$ by $\boldsymbol{\epsilon}_{J / \psi}^{* T} \cdot \boldsymbol{\epsilon}_{\phi}^{* T}$, contributing only to $A_{\|}$, where the superscripts $T$ refer to projections perpendicular to $\hat{\mathbf{p}}$. The CP-odd amplitude $\boldsymbol{\epsilon}_{J / \psi}^{*} \times \boldsymbol{\epsilon}_{\phi}^{*} \cdot \hat{\mathbf{p}}$ contributes only to $A_{\perp}$. The case of transverse (\| or $\perp$ ) polarization states is reminiscent of photon polarization correlations [17] in neutral pion decay. Thus we may write the decay amplitude as

$$
A\left(B_{s} \rightarrow J / \psi \phi\right)=A_{0}\left(m_{\phi} / E_{\phi}\right) \boldsymbol{\epsilon}_{J / \psi}^{* L} \boldsymbol{\epsilon}_{\phi}^{* L}-A_{\|} \boldsymbol{\epsilon}_{J / \psi}^{* T} \cdot \boldsymbol{\epsilon}_{\phi}^{* T} / \sqrt{2}-i A_{\perp} \boldsymbol{\epsilon}_{J / \psi}^{*} \times \boldsymbol{\epsilon}_{\phi}^{*} \cdot \hat{\mathbf{p}} / \sqrt{2},
$$

where $E_{\phi}$ is the energy of the $\phi$ in the $J / \psi$ rest frame, and the individual amplitudes are real in the absence of final-state interactions. The amplitudes for the corresponding decays of $\bar{B}_{s} \equiv C P\left(B_{s}\right)$ are $\bar{A}_{0}=A_{0}, \bar{A}_{\|}=A_{\|}$, and $\bar{A}_{\perp}=-A_{\perp}$. (We can see directly by counting powers of $\hat{\mathbf{p}}$ that $A_{0}$ and $A_{\|}$are P-even while $A_{\perp}$ is $\mathrm{P}$-odd.) We have normalized the partial widths for the three independent polarization states in such a way that

$$
d \Gamma\left(B_{s} \rightarrow J / \psi \phi\right) / d t=\left|A_{0}\right|^{2}+\left|A_{\|}\right|^{2}+\left|A_{\perp}\right|^{2},
$$


and we may identify

$$
p(t)=\left|A_{0}\right|^{2}+\left|A_{\|}\right|^{2} \quad, \quad m(t)=\left|A_{\perp}\right|^{2} .
$$

For purposes of comparing with other notations, we can express the helicity amplitudes $A_{\lambda}$ (where $\lambda=1,0,-1$ is the projection of the $\phi$ angular momentum on the $x$ axis) in terms of the linear polarization basis by $A_{ \pm 1}=\left(A_{\|} \pm A_{\perp}\right) / \sqrt{2}$, with $A_{0}$ the same in either basis, and in terms of $\mathrm{S}-, \mathrm{P}-$, and $\mathrm{D}$-wave amplitudes by

$$
A_{ \pm 1}=\sqrt{\frac{1}{3}} S \pm \sqrt{\frac{1}{2}} P+\sqrt{\frac{1}{6}} D \quad, \quad A_{0}=-\sqrt{\frac{1}{3}} S+\sqrt{\frac{2}{3}} D .
$$

With these normalizations,

$$
d \Gamma\left(B_{s} \rightarrow J / \psi \phi\right) / d t=\left|A_{0}\right|^{2}+\left|A_{1}\right|^{2}+\left|A_{-1}\right|^{2}=|S|^{2}+|P|^{2}+|D|^{2} \quad,
$$

and

$$
A_{\|}=\sqrt{\frac{2}{3}} S+\sqrt{\frac{1}{3}} D \quad, \quad A_{\perp}=P
$$

The longitudinal and transverse partial widths are given, respectively, by

$$
d \Gamma_{0} / d t=\left|A_{0}\right|^{2} \quad, \quad d \Gamma_{T} / d t=\left|A_{1}\right|^{2}+\left|A_{-1}\right|^{2} \quad .
$$

In terms of partial-wave amplitudes, one has

while

$$
\begin{gathered}
\frac{d \Gamma_{0}}{d t}=|-\sqrt{1 / 3} S+\sqrt{2 / 3} D|^{2} \quad, \quad \frac{d \Gamma_{T}}{d t}=|\sqrt{2 / 3} S+\sqrt{1 / 3} D|^{2}+|P|^{2} \\
\frac{d \Gamma_{\|}}{d t}=|\sqrt{2 / 3} S+\sqrt{1 / 3} D|^{2}, \frac{d \Gamma_{\perp}}{d t}=|P|^{2}
\end{gathered}
$$

$$
\frac{p(t)}{p(t)+m(t)}=\frac{|S|^{2}+|D|^{2}}{|S|^{2}+|P|^{2}+|D|^{2}} \quad ; \quad \frac{m(t)}{p(t)+m(t)}=\frac{|P|^{2}}{|S|^{2}+|P|^{2}+|D|^{2}} \quad .
$$

Finally, we note that in the covariant expression [18]

$$
A_{\lambda}=\epsilon_{1 \mu}^{*} \epsilon_{2 \nu}^{*}\left[a g^{\mu \nu}+\frac{b}{m_{1} m_{2}} p_{2}^{\mu} p_{1}^{\nu}+\frac{i c}{m_{1} m_{2}} \epsilon^{\mu \nu \alpha \beta} p_{1 \alpha} p_{2 \beta}\right]
$$

for the decay $B \rightarrow V_{1} V_{2}$, where $\epsilon^{0123} \equiv+1$ and $V_{1}$ and $V_{2}$ are vector mesons with masses $m_{1}$ and $m_{2}$ and four-momenta $p_{1}$ and $p_{2}$, the helicity amplitudes are

$$
A_{ \pm 1}=a \pm c \sqrt{x^{2}-1} \quad, \quad A_{0}=-a x-b\left(x^{2}-1\right)
$$

where $x \equiv p_{1} \cdot p_{2} /\left(m_{1} m_{2}\right)$. We thus identify

$$
S=\frac{1}{\sqrt{3}}\left[a(2+x)+b\left(x^{2}-1\right)\right], P=c \sqrt{2\left(x^{2}-1\right)}, \quad D=\sqrt{\frac{2}{3}}\left[a(1-x)-b\left(x^{2}-1\right)\right] .
$$


Note that $S$ and $D$ both involve $a$ and $b$.

The derivation of Eq. (3) is elementary. The $\phi$ is coupled to $K^{+} K^{-}$through an amplitude $\epsilon_{\phi} \cdot\left(p_{K^{+}}-p_{K^{-}}\right)$, where the quantities denote 4 -vectors. Thus the plane of (linear) $\phi$ polarization is related to that of the $K^{+} K^{-}$system in the $J / \psi$ rest frame. By definition, we have taken the $\phi$ linear polarization vector to lie in the $x-y$ plane. We may define an angle $\psi$ as that of the $K^{+}$in the $\phi$ rest frame relative to the helicity axis (the negative of the direction of the $J / \psi$ in that frame). The spatial components of the $\phi$ and $J / \psi$ polarizations must be correlated since the decaying strange $B$ is spinless. The $J / \psi$ then has a single linear polarization state $\boldsymbol{\epsilon}$ for each amplitude: In the $J / \psi$ rest frame,

$$
A_{\|}: \quad \boldsymbol{\epsilon}=\hat{y} \quad ; \quad A_{0}: \quad \boldsymbol{\epsilon}=\hat{x} \quad ; \quad A_{\perp}: \quad \boldsymbol{\epsilon}=\hat{z}
$$

A unit vector $n$ in the direction of the $\ell^{+}$in $J / \psi$ decay may be defined to have components

$$
\left(n_{x}, n_{y}, n_{z}\right)=(\sin \theta \cos \varphi, \sin \theta \sin \varphi, \cos \theta)
$$

where $\varphi$ is the angle between the projection of the $\ell^{+}$on the $K^{+} K^{-}$plane in the $J / \psi$ rest frame and the $x$ axis. The sum over lepton polarizations then leads to a tensor in the $J / \psi$ rest frame with spatial components (in the limit of zero lepton mass, assumed here)

$$
\sum_{\ell^{ \pm} \text {pol }}\left[\bar{u} \gamma_{i} v\right]^{*}\left[\bar{u} \gamma_{j} v\right] \sim L_{i j} \equiv \delta_{i j}-n_{i} n_{j}
$$

Physically this tensor simply expresses the fact that massless lepton pairs couple only to transverse polarization states of the $J / \psi$, as expected from the structure of the electromagnetic interactions.

Taking account of the definition (6), we then find that the probability for the decay $B_{s} \rightarrow\left(\ell^{+} \ell^{-}\right)_{J / \psi}\left(K^{+} K^{-}\right)_{\phi}$ is proportional to

$$
\sum_{\ell^{ \pm} \text {pol }}|A|^{2}=A_{i} A_{j}^{*} L_{i j}
$$

where

$$
A_{i}=A_{0} \delta_{i x} \cos \psi-A_{\|} \delta_{i y} \sin \psi / \sqrt{2}+i A_{\perp} \delta_{i z} \sin \psi / \sqrt{2}
$$

Consequently, when we use the definitions (19), we find

$$
\begin{gathered}
\quad \frac{d^{4} \Gamma\left[B_{s} \rightarrow\left(\ell^{+} \ell^{-}\right)_{J / \psi}\left(K^{+} K^{-}\right)_{\phi}\right]}{d \cos \theta d \varphi d \cos \psi d t}=\frac{9}{32 \pi}\left[2\left|A_{0}\right|^{2} \cos ^{2} \psi\left(1-\sin ^{2} \theta \cos ^{2} \varphi\right)\right. \\
+\sin ^{2} \psi\left\{\left|A_{\|}\right|^{2}\left(1-\sin ^{2} \theta \sin ^{2} \varphi\right)+\left|A_{\perp}\right|^{2} \sin ^{2} \theta-\operatorname{Im}\left(A_{\|}^{*} A_{\perp}\right) \sin 2 \theta \sin \varphi\right\} \\
\left.+\frac{1}{\sqrt{2}} \sin 2 \psi\left\{\operatorname{Re}\left(A_{0}^{*} A_{\|}\right) \sin ^{2} \theta \sin 2 \varphi+\operatorname{Im}\left(A_{0}^{*} A_{\perp}\right) \sin 2 \theta \cos \varphi\right\}\right] .
\end{gathered}
$$

The overall normalization has been chosen to agree with our previous conventions when one integrates over angles. For $\bar{B}_{s}$ decays the interference terms involving $A_{\perp}$ amplitudes are of opposite sign and all other terms are unchanged. 
Integration over $\cos \psi$ leads to the distribution

$$
\begin{gathered}
\frac{d^{3} \Gamma\left[B_{s} \rightarrow\left(\ell^{+} \ell^{-}\right)_{J / \psi}\left(K^{+} K^{-}\right)_{\phi}\right]}{d \cos \theta d \varphi d t}=\frac{3}{8 \pi}\left[\left|A_{0}\right|^{2}\left(1-\sin ^{2} \theta \cos ^{2} \varphi\right)+\left|A_{\|}\right|^{2}\left(1-\sin ^{2} \theta \sin ^{2} \varphi\right)\right. \\
\left.+\left|A_{\perp}\right|^{2} \sin ^{2} \theta-\operatorname{Im}\left(A_{\|}^{*} A_{\perp}\right) \sin 2 \theta \sin \varphi\right] .
\end{gathered}
$$

Performing the integrals over $\varphi$ and taking account of the differing time-dependences of the decays of $B_{s}^{( \pm)}$, we obtain the result (3). This is a suitable single-angle distribution to employ if one wishes to disentangle the CP-even and CP-odd components of the $B_{s}$. The two-angle distribution (24) allows one to separate out the individual quantities $\left|A_{0}\right|^{2}$, $\left|A_{\|}\right|^{2}$, and $\left|A_{\perp}\right|^{2}$.

An interesting oscillation appears in the interference terms between CP-even and CPodd decays. For example, in the two-angle distribution (24), since the respective timedependences of the $B_{s}^{(+)}$and $B_{s}^{(-)}$decay amplitudes are $e^{-i m_{L} t-\Gamma_{L} t / 2}$ and $e^{-i m_{H} t-\Gamma_{H} t / 2}$, the term $-\operatorname{Im}\left(A_{\|}^{*} A_{\perp}\right)$ behaves as $\left|A_{\|}(0) A_{\perp}(0)\right| \sin (\Delta m t-\delta) e^{-\bar{\Gamma} t}$, where $\delta$ is a strong final-state phase shift difference: $A_{\|}(0)^{*} A_{\perp}(0)=\left|A_{\|}(0) A_{\perp}(0)\right| e^{i \delta}$. Thus, if one tags the flavor of the decaying $B_{s}$, one can observe the effects of $\Delta m$ in the $J / \psi \phi$ final state through the interference of final states of opposite CP (if both are present). The oscillation term averages out to zero if the initial numbers of $B_{s}$ and $\bar{B}_{s}$ are equal.

The distributions (3), (23), and (24) also permit one to separate out the components $\left|A_{0}\right|^{2},\left|A_{\|}\right|^{2},\left|A_{\perp}\right|^{2}$, and the interference terms $\operatorname{Im}\left(A_{\|}^{*} A_{\perp}\right), \operatorname{Re}\left(A_{0}^{*} A_{\|}\right)$, and $\operatorname{Im}\left(A_{0}^{*} A_{\perp}\right)$ for the decays $B^{0} \rightarrow J / \psi K^{* 0}$. (Here and subsequently we imply the sum over a process and its charge-conjugate.) Moreover, in the limit of flavor $\mathrm{SU}(3)$ symmetry, one expects the ratios of the relative components in $B^{0} \rightarrow J / \psi K^{* 0}$ to be the same as those at proper time $t=0$ in the decays $B_{s} \rightarrow J / \psi \phi[19]$. Thus, an analysis of $B^{0} \rightarrow J / \psi K^{* 0}$ can provide an independent estimate of the relative contributions of CP-even and CP-odd final states at $t=0$ to the decays $B_{s} \rightarrow J / \psi \phi$, enhancing the ability to determine $\Gamma_{H}$ and $\Gamma_{L}$.

If the $K^{*}$ is observed to decay to the CP eigenstate $K_{S} \pi^{0}$, the amplitudes $A_{0}$ and $A_{\|}$refer (as in the case of $J / \psi \phi$ ) to the CP-even eigenstate, while $A_{\perp}$ refers to the CPodd eigenstate. The expected dominance of the CP-even eigenstate (see below) means that in $B^{0} \rightarrow J / \psi K_{S} \pi^{0}$ events the $\mathrm{CP}$ asymmetry will tend to be opposite to that in $B^{0} \rightarrow J / \psi K_{S}$ [12, 20]. Since the rates for observing the processes $B^{0} \rightarrow J / \psi K_{S}$ and $B^{0} \rightarrow J / \psi K^{* 0} \rightarrow J / \psi K_{S} \pi^{0}$ are comparable (taking account of branching ratios and typical detection efficiencies), the incorporation of $J / \psi K_{S} \pi^{0}$ data may add statistical power to any experiment studying the $J / \psi K_{S}$ final state, even when the $\pi^{0}$ is not observed directly but its existence inferred.

The distribution (3) permits one to separate amplitudes of opposite parity from one another even if the $K^{+} K^{-}$system in $B_{s} \rightarrow J / \psi K^{+} K^{-}$or the $K \pi$ system in $B \rightarrow J / \psi K \pi$ is not a vector meson [12]. This is easily seen by considering the density matrix $\rho_{i j}$ of the $J / \psi$, expressed in terms of linear polarization states, so that the decay rate is proportional to $\rho_{i j} L_{i j}$, with $L_{i j}$ defined in (20). If we integrate over $\varphi$, we find

$$
\frac{d^{2} \Gamma}{d \cos \theta d t} \sim\left(\rho_{x x}+\rho_{y y}\right)\left(\frac{1+\cos ^{2} \theta}{2}\right)+\rho_{z z} \sin ^{2} \theta \text {, }
$$


where $\rho_{x x}$ and $\rho_{y y}$ correspond to linear $J / \psi$ polarization states in the plane of the two pseudoscalar mesons, while $\rho_{z z}$ corresponds to $J / \psi$ polarization perpendicular to this plane, and thus represents an amplitude with parity opposite to those contributing to $\rho_{x x}$ and $\rho_{y y}$. For cases where each particle in the final state is a $\mathrm{C}$ eigenstate as in $B^{0} \rightarrow J / \psi K^{* 0} \rightarrow J / \psi K_{S} \pi^{0}$ and $B_{s} \rightarrow J / \psi \phi \rightarrow J / \psi K_{S} K_{L}$ the parity separation is also a CP separation and the transversity analysis can be used without the need to extract the vector resonance from nonresonant or other background.

The dominance of the $\left|A_{0}\right|^{2}$ contribution in $B^{0} \rightarrow J / \psi K^{* 0}$ decays [14, 21, 22] implies via flavor SU(3) that the $\left|A_{0}\right|^{2}$ contribution should also dominate $B_{s} \rightarrow J / \psi \phi$, and hence that $B_{s}^{(-)} \rightarrow J / \psi \phi$ is likely to be suppressed in comparison with $B_{s}^{(+)} \rightarrow J / \psi \phi$. Thus the initial angular distribution is very likely to be dominated by the $1+\cos ^{2} \theta$ component. As time increases, the fraction of the angular distribution proportional to this component will decrease while that proportional to $\sin ^{2} \theta$ will increase. It should be possible to separate out the two components by a combined analysis in $\theta$ and proper decay time. If the $\sin ^{2} \theta$ component does not show up even at large times, a single-exponential fit to the decay should provide a good estimate of the lifetime of the CP-even eigenstate.

The angular distribution in (3) has the form of an ellipsoid which is prolate if $p(t)>$ $2 m(t)$ and oblate if $p(t)<2 m(t)$. (Here we imagine an average over $\varphi$ to have been performed.) If all three partial waves are equally populated $p(t)=2 m(t)$ since there are two partial waves with even $\mathrm{CP}$ and only one with odd CP. For this case the angular distribution is isotropic in $\cos \theta$ as expected.

If $p(0)>2 m(0)$ (i.e., if the CP-even decay is initially more than $2 / 3$ dominant), if the $\mathrm{CP}$-even eigenstate $B_{s}^{(+)}$has the greater decay rate as expected, and if there is a nonzero odd-CP component $m(0) \neq 0$, then the angular distribution in transversity will be initially prolate but will eventually become oblate as the quantity $p(t)-2 m(t)$ changes sign. This effect can be noted by dividing the events into two bins with $|\cos \theta|<1 / 2$ and $|\cos \theta|>1 / 2$, denoted by $E$ (equatorial) and $P$ (polar) respectively:

$$
\begin{gathered}
E \equiv \int_{-1 / 2}^{1 / 2} d(\cos \theta) \frac{d^{2} \Gamma}{d \cos \theta d t}=\frac{13}{32} p(t)+\frac{11}{16} m(t) \\
P \equiv\left[\int_{-1}^{-1 / 2}+\int_{1 / 2}^{1}\right] d(\cos \theta) \frac{d^{2} \Gamma}{d \cos \theta d t}=\frac{19}{32} p(t)+\frac{5}{16} m(t) \\
P-E=\frac{3}{16}[p(t)-2 m(t)]
\end{gathered}
$$

The difference between the numbers in the two bins provides an experimental number whose sign will change in time under the assumptions noted above. This two-bin analysis of data can be adjusted for optimum statistics by changing the sizes of the bins to correspond to the initial values of $p(0)$ and $m(0)$.

The analysis performed by CDF [14] for $B_{s} \rightarrow J / \psi \phi$ was also based on a singleangle distribution, but it separated out the transverse component from the longitudinal component. In the absence of vertex cuts these would be, respectively, $\Gamma_{T} \equiv$ $\int_{0}^{\infty} d t\left(\left|A_{\|}\right|^{2}+\left|A_{\perp}\right|^{2}\right)$ and $\Gamma_{0} \equiv \int_{0}^{\infty} d t\left(\left|A_{0}\right|^{2}\right)$. With a minimum vertex cut of $50 \mu \mathrm{m}$, the 
result obtained was $\Gamma_{0} /\left(\Gamma_{0}+\Gamma_{T}\right)=0.56 \pm 0.21$ (stat) ${ }_{-0.04}^{+0.02}$ (sys). The transverse component contains both $\mathrm{CP}$-even and $\mathrm{CP}$-odd contributions, while the longitudinal component is CP-even. The separation of transverse and longitudinal components makes sense only if the $B_{s}^{(-)} \rightarrow J / \psi \phi$ decay is negligible, or if the lifetime difference between $B_{s}^{(+)}$and $B_{s}^{(-)}$can be ignored.

Corresponding determinations of $\Gamma_{0} /\left(\Gamma_{0}+\Gamma_{T}\right)$ for the decay $B^{0} \rightarrow J / \psi K^{* 0}$ are $0.65 \pm 0.10 \pm 0.04(\mathrm{CDF})$ [14], $0.97 \pm 0.16 \pm 0.15$ (ARGUS) [21], $0.80 \pm 0.08 \pm 0.05$ (CLEO) [22], and $0.74 \pm 0.07$ (world average) [14]. This last value is compatible with the corresponding one for $B_{s} \rightarrow J / \psi \phi$. A discrepancy would have indicated either a violation of $\mathrm{SU}(3)$ or the lifetime effect mentioned above.

The presence of two eigenstates with possibly differing lifetimes can affect any determination of $\tau\left(B_{s}\right)$. When observing the $B_{s}$ in a final state of definite flavor, such as $D_{s} \pi$ or $D_{s} \ell \nu_{\ell}$, one will be effectively measuring the average lifetime $\bar{\Gamma}$ of the CP-even and CP-odd states. Most measurements reported up to now, including a recent CDF determination [23] leading to a world average [24] of $\tau\left(B_{s}\right)=1.58 \pm 0.10 \mathrm{ps}$, are of this type. This quantity is expected [4] to be very close to the $B^{0}$ lifetime, for which the world average [24] is $\tau\left(B^{0}\right)=1.57 \pm 0.05$ ps. However, minimum-lifetime cuts can bias the sample against the CP-even (shorter-lived) component, leading to results which depend on the cut if a single-exponential fit is adopted.

To summarize, we have found that a combined analysis with respect to proper decay time and a single transversity angle in the decay $B_{s} \rightarrow J / \psi \phi$ can determine the lifetime of at least the CP-even and possibly the CP-odd mass eigenstates of the $B_{s}-\bar{B}_{s}$ system. Additional information about the properties of the $J / \psi \phi$ mode at proper time $t=0$ can be obtained by a similar analysis of the decays $B^{0} \rightarrow J / \psi K^{* 0}$. These analyses can already be attempted with the data sample [14] now in hand.

We thank B. Kayser, R. Kutschke, K. Ohl, M. Paulini, E. A. Paschos, M. P. Schmidt, and W. Wester for useful discussions. This work was supported in part by the United States Department of Energy under Contracts No. DE AC02 76CH03000, DE FG02 90ER40560 and W-31-109-ENG-38. 


\section{References}

[1] N. Cabibbo, Phys. Rev. Lett. 10 (1963) 531; M. Kobayashi and T. Maskawa, Prog. Theor. Phys. 49 (1973) 652.

[2] See, e.g., J. L. Rosner, Enrico Fermi Institute Report No. EFI 95-36, hepph/9506364, lectures presented at the VIII J. A. Swieca Summer School, Rio de Janeiro, Feb. 7-11, 1995, proceedings to be published by World Scientific.

[3] R. Aleksan, A. Le Yaouanc, L. Oliver, O. Pène, and J. C. Raynal, Phys. Lett. B 316 (1993) 567.

[4] M. B. Voloshin, N. G. Uraltsev, V. A. Khoze, and M. A. Shifman, Yad. Fiz. 46 (1987) 181 [Sov. J. Nucl. Phys. 46 (1987) 112]; I. I. Bigi et al., in B Decays, edited by S. Stone (World Scientific, Singapore, 1994), p. 132; I. I. Bigi, Univ. of Notre Dame Report No. UND-HEP-95-BIG02, June, 1995, to be published in Physics Reports.

[5] A. Soni, Brookhaven National Laboratory report BNL-62284, October, 1995, to be published in Proceedings of Lattice 95, Melbourne, Australia, July 10-13, 1995.

[6] I. Dunietz, Phys. Rev. D 52 (1995) 3048.

[7] G. Buchalla, I. Dunietz, and H. Yamamoto, FERMILAB-PUB-95/167-T, July, 1995, to be published in Phys. Lett. B.

[8] T. E. Browder and S. Pakvasa, Phys. Rev. D 52 (1995) 3123.

[9] L. L. Chau, W.-Y. Keung, and M. D. Tran, Phys. Rev. D 27 (1983) 2145; L. L. Chau, Phys. Rep. 95 (1983) 1; A. J. Buras, W. Slominsky, and H. Steger, Nucl. Phys. B245 (1984) 369; A. Datta, E. A. Paschos, and U. Türke, Phys. Lett. B 196 (1987) 382; A. Datta, E. A. Paschos, and Y. L. Wu, Nucl. Phys. B311 (1988) 35; I. Dunietz, Ann. Phys. (N.Y.) 184 (1988) 350.

[10] N.-P. Chang and C. A. Nelson, Phys. Rev. Lett. 40 (1978) 1617; Phys. Rev. D 20 (1978) 2923; C. A. Nelson, Phys. Rev. D 30 (1984) 107, 1937; 32 (1985) 1848(E).

[11] H. J. Lipkin, in Proceedings of the SLAC Workshop on Physics and Detector Issues for a High-Luminosity Asymmetric B Factory, edited by David Hitlin, published as SLAC, LBL and Caltech reports SLAC-373, LBL-30097 and CALT-68-1697, 1990, p. 49.

[12] I. Dunietz, H. R. Quinn, A. Snyder, W. Toki and H. J. Lipkin, Phys. Rev. D 43 (1991) 2193.

[13] I. Dunietz, Appendix in B Decays, edited by S. Stone (World Scientific, Singapore, 1994), p. 550. 
[14] CDF Collaboration, F. Abe et al., Phys. Rev. Lett. 75 (1995) 3068.

[15] A. Bohr, Nucl. Phys. 10 (1959) 486.

[16] J. L. Rosner, Phys. Rev. D 42 (1990) 3732.

[17] L. D. Landau, Dokl. Akad. Nauk SSSR 60 (1948) 207; C. N. Yang, Phys. Rev. 77 (1950) 242, 722.

[18] G. Kramer and W. F. Palmer, Phys. Rev. D 45 (1992) 193; 46 (1992) 2969, 3197; Zeit. Phys. C 55 (1992) 497.

[19] I. Dunietz, in Proceedings of the Workshop on B Physics at Hadron Accelerators, Snowmass, Colorado, June 21 - July 2, 1993, edited by P. McBride and C. S. Mishra (Fermilab report Fermilab-CONF-93/267, 1993), p. 83.

[20] B. Kayser, M. Kuroda, R. D. Peccei, and A. I. Sanda, Phys. Lett. B 237 (1990) 508.

[21] ARGUS Collaboration, H. Albrecht et al., Phys. Lett. B 340 (1994) 217.

[22] CLEO Collaboration, M. S. Alam et al., Phys. Rev. D 50 (1994) 43.

[23] CDF Collaboration, F. Abe et al., Phys. Rev. Lett. 74 (1995) 4988.

[24] I. J. Kroll, presented at 17th International Symposium on Lepton and Photon Interactions, Beijing, 11 August 1995. 